\title{
ANÁLISE DO DESENVOLVIMENTO MUNICIPAL PARANAENSE: UMA ABORDAGEM ESPACIAL PARA A DÉCADA DE 2000
}

\author{
Vinicius Misael Alves de Lima ${ }^{1}$ \\ Carlos Eduardo Caldarelli ${ }^{2}$ \\ Marcia Regina Gabardo da Camara ${ }^{3}$
}

\begin{abstract}
Resumo
O objetivo deste estudo é analisar o desenvolvimento municipal paranaense, nos anos de 2000, 2005 e 2010, e verificar a presença de autocorrelação espacial e a formação de clusters de desenvolvimento entre os seus municípios. Como indicador de desenvolvimento foi utilizado o Índice FIRJAN de Desenvolvimento Municipal - IFDM, considerando: o índice agregado e os sub-índices Emprego \& Renda, Educação e Saúde. Realizou-se a análise estatística descritiva da evolução do IFDM e a Análise Exploratória de Dados Espaciais (AEDE) para verificar a existência de correlação espacial e de clusters de cidades desenvolvidas e não desenvolvidas. $O$ presente estudo permite concluir que o desenvolvimento dos municípios paranaenses apresentou melhora para o Estado e para a maioria dos municípios, tanto para o índice geral como para os sub-índices de Emprego \& Renda, Educação e Saúde. No que tange à análise espacial dos dados constatou-se que existe autocorrelação espacial e ela é positiva para todos os anos estudados. Nos três anos analisados, os clusters de desenvolvimento se distribuíram basicamente em três mesorregiões: Norte Central, Oeste, e Metropolitana de Curitiba. Os clusters de subdesenvolvimento se distribuíram na região central e leste do estado.
\end{abstract}

Palavras-chave: IFDM; desenvolvimento; Análise Espacial; Paraná

\begin{abstract}
The aim of this study is to analyze Paraná 's municipal development in the years 2000, 2005 and 2010, to verify the presence of spatial autocorrelation and cluster formation among its municipalities. As development indicator index FIRJAN Municipal Development - IFDM was used considering the aggregate: employ / income, education and health. Carried out the descriptive statistical analysis of the evolution of IFDM and Exploratory Spatial Data Analysis (ESDA) to verify the existence of spatial correlation and identification of cities' cluster developed and undeveloped. The present study concluded that the municipal development, using the data from the IFDM, showed improvement, not only in the State but also in most municipalities, both for the overall index Education and Health. Regarding the spatial analysis of the data it was found that spatial autocorrelation exists and it is positive for all years studied. In the three years analyzed, clusters development is distributed in three regions: North Central, West, and Metropolitan Curitiba. Clusters of underdevelopment were distributed in central and eastern state.
\end{abstract}

Keywords: IFDM; development; Spatial Analysis; Paraná

\footnotetext{
${ }^{1}$ Mestrando do Programa de Pós-Graduação de Mestrado em Economia Regional - UEL. E-mail: viniciusmisael@gmail.com

${ }^{2}$ Professor do Departamento de Economia da Universidade Estadual de Londrina - UEL. E-mail: carlos.caldarelli@gmail.com

${ }^{3}$ Professora do Departamento de Economia da Universidade Estadual de Londrina - UEL. E-mail: mgabardo@sercomtel.com.br
}

Recebido em: 17/10/2013

Aceito em: 14/01/2014 


\section{Introdução}

Estudos que enfoquem o desenvolvimento econômico têm sido bastante discutidos na ciência econômica, nos últimos anos. Segundo Todaro apud Leite (1983), o desenvolvimento econômico pode ser entendido como um processo de melhoria na qualidade de vida das pessoas. Nas ciências sociais, em específico, define-se o desenvolvimento como uma melhora qualitativa no nível de bem-estar de um determinado grupo social, usando-se como referência os indicadores sociais, políticos, culturais e econômicos disponíveis.

A mensuração do nível de desenvolvimento de uma localidade é uma tarefa difícil, pois envolve a variação no produto per capita e uma série de melhorias nos indicadores econômicos e sociais de uma determinada localidade. Dada complexidade que abarca o conceito, ao longo dos anos, indicadores compostos vêm sendo criados para mensurá-lo.

Uma iniciativa da Federação das Indústrias do Rio de Janeiro (FIRJAN) foi a criação do Índice FIRJAN de Desenvolvimento Municipal (IFDM). O índice é composto por três áreas importantes para o desenvolvimento: Emprego \& Renda, Educação e Saúde e utiliza-se exclusivamente de estatísticas públicas oficiais. O IFDM se distingue por sua periodicidade anual e por acompanhar o desenvolvimento de todos os 5.565 municípios brasileiros.

No que tange ao estado do Paraná, por meio do IFDM é possível identificar municípios que apresentam um bom desempenho em relação ao desenvolvimento, assim como municípios subdesenvolvidos. Essas informações quando submetidas a uma análise espacial, podem mostrar quais regiões particulares do estado apresentam concentrações de desenvolvimento ou subdesenvolvimento. Assim, será possível responder a seguinte pergunta: como se encontra distribuído espacialmente o desenvolvimento no estado do Paraná?

O presente trabalho tem por objetivo analisar desenvolvimento dos municípios do estado do Paraná para os anos de 2000, 2005 e 2010, e o padrão espacial de desenvolvimento no estado, para tanto, busca-se verificar a presença de autocorrelação espacial e a possível formação de clusters entre os seus municípios. A pesquisa se justifica pela relativa escassez de estudos com enfoque em desenvolvimento e abordagem espacial para o Paraná, sobretudo investigando índices de desenvolvimento. Por fim, destaca-se a importância do estudo na busca de melhor entender o padrão de desenvolvimento no estado, servindo desta forma como suporte para possíveis políticas governamentais.

Além desta introdução, o presente estudo divide-se em mais quatro seções. A seção 2 faz uma revisão da literatura empírica da análise espacial aplicada ao desenvolvimento. $\mathrm{Na}$ terceira são apresentados os procedimentos metodológicos adotados. As discussões e os 
resultados obtidos são apresentados na seção 4. Finalmente, a seção 5 apresenta as conclusões deste estudo.

\section{Revisão de Literatura}

A Análise Exploratória de Dados Espaciais ganhou destaque recentemente, entre outros fatores, devido ao avanço da capacidade computacional. No Brasil, os estudos utilizando tais técnicas tem se desenvolvimento em diversas áreas e aplicações, todavia, no que concerne à análise espacial e desenvolvimento econômico a literatura ainda é escassa.

Costa et. al. (2007) analisaram a dependência espacial do desenvolvimento humano no estado do Rio Grande do Norte, verificando o comportamento do Índice de Desenvolvimento Humano Municipal (IDH-M) em suas três dimensões: educação, longevidade e renda per capita. Os aludidos autores buscaram verificar a existência de clusters, padrões ou tendências de desenvolvimento e/ou de pobreza no estado. Os resultados mostraram que existe uma dependência espacial nos municípios do RN para os indicadores do IDHM e suas dimensões (Educação, Longevidade e Renda), sendo confirmado que a localização geográfica possui um importante papel no desenvolvimento humano do estado.

Lorena et. al. (2011), baseados no estudo de Costa et. al. (2007), analisaram a dependência e/ou semelhança espacial entre os municípios do estado do Espírito Santo, utilizando como variável o IDH-M e as dimensões: educação, expectativa de vida, renda e PIB. Os resultados revelaram a existência de dependência espacial para as dimensões Longevidade, Renda e PIB no Espírito Santo.

Gama e Strauch (2009) efetuaram um estudo nos municípios da Bacia Hidrográfica do Paraíba do Sul com o objetivo de construir um índice sintético de desenvolvimento sustentável baseado nas dimensões social, econômica, ambiental e institucional. Depois de construído o índice aplicou-se a análise espacial para identificar clusters de desenvolvimento e sustentabilidade entre os municípios.

Vale e Silva (2011) obtiveram um índice de ruralidade utilizando a técnica multivariada de análise fatorial. Calculado esse índice os autores utilizaram a análise espacial para determinar estruturas de autocorrelação espacial em todo o Nordeste e entre os municípios, permitindo a identificação de clusters e outliers. Os resultados mostram uma tendência à formação de clusters no Nordeste. 


\section{Metodologia}

\subsection{Análise Exploratória de Dados Espaciais}

Os índices de desenvolvimento de cada município do Paraná foram georeferenciados para que, por meio da Análise Exploratória de Dados Espaciais (AEDE), seja possível verificar padrões de correlação espacial entre as variáveis e assim identificar concentrações de desenvolvimento e subdesenvolvimento.

A primeira lei da geografia diz que tudo está relacionado com tudo, mas elementos mais próximos estão mais relacionados que elementos mais distantes (Tobler apud Asselin, 1993). Dessa forma, a AEDE procura descrever padrões de correlação espacial entre os dados, ou sobre a presença de agrupamentos (clusters) nos dados, ou, ainda, sobre a influência de observações discrepantes (outliers). Segundo Almeida (2004) o objetivo primordial da AEDE é deixar os dados espaciais falarem por eles próprios.

\subsubsection{Matriz de Pesos Espaciais}

Para proceder a AEDE é necessária a criação de uma matriz de pesos espaciais $\left(w_{i j}\right)$, que é a forma de expressar a estrutura espacial dos dados. A matriz contém a informação de quanto a interação é mais forte em municípios mais próximos, e mais fraca em municípios mais distantes.

Moran (1948) e Geary (1954) desenvolveram a noção de contiguidade binária entre unidades espaciais. De acordo com essa noção, a estrutura de vizinhança subjacente seria expressa por uma matriz binária simétrica, em que se atribui 1 para municípios que são vizinhos e 0 para municípios que não possuem vizinhança. Formalmente, temos:

$$
w_{i j}= \begin{cases}1, & \text { se } i \text { e } j \text { são vizinhos } \\ 0, & \text { se } i \text { e } j \text { não são vizinhos }\end{cases}
$$

Apesar de parecer simples, a definição de uma vizinhança envolve várias possibilidades, conforme distintas convenções de vizinhança.

\begin{tabular}{|c|c|c|}
\hline & $\mathrm{b}$ & \\
\hline $\mathrm{b}$ & $\mathrm{a}$ & $\mathrm{b}$ \\
\hline & $\mathrm{b}$ & \\
\hline \multicolumn{3}{|c|}{ rook }
\end{tabular}

\begin{tabular}{|c|c|c|}
\hline $\mathrm{c}$ & $\mathrm{b}$ & $\mathrm{c}$ \\
\hline $\mathrm{b}$ & $\mathrm{a}$ & $\mathrm{b}$ \\
\hline $\mathrm{c}$ & $\mathrm{b}$ & $\mathrm{c}$ \\
\hline \multicolumn{3}{|c}{ queen }
\end{tabular}

Figura 1 - Tipos de vizinhança nas unidades espaciais.

Fonte: Elaborado pelo autor, a partir de Anselin (1988). 
As convenções mais utilizadas na literatura são a torre (rook) e a rainha (queen). A diferença entre as duas convenções é que a rook não considera os vértices como regiões de tangência, enquanto a queen considera (Figura 1). Nesse trabalho a convenção utilizada será a queen.

\subsection{2. "I" de Moran}

Construída a matriz de pesos espaciais ${ }^{4}$, é necessário verificar se há uma estrutura de autocorrelação espacial nos dados. Moran (1948) propôs uma estatística muito usada atualmente, o I de Moran. Em notação matricial ele pode ser expresso como:

$$
I=\frac{n}{S_{o}}\left(\mathbf{z}^{\prime} \mathbf{z}\right)^{-1} \mathbf{z}^{\prime} \mathbf{W} \mathbf{z}
$$

Onde $n$ é um escalar que representa o número de unidades espaciais, $\mathbf{z}$ é um vetor de desvios da variável de interesse em relação a sua média ${ }^{5}, \mathbf{W}$ é a matriz de pesos espaciais e $S_{0}$ é um escalar que representa a soma dos elementos de W. Como $S_{0}=\sum_{i=1}^{n} \sum_{j=1}^{n} w_{i j}$, no caso de uma matriz padronizada para a soma de linha igualar a $1, \sum_{j=1}^{n} w_{i j}=1$ e portanto, $S_{0}=\sum_{i=1}^{n} 1=n$. Assim, o termo $\frac{n}{S_{0}}$ simplifica para 1 e a estatística I pode ser escrita como:

$$
I=\left(\mathbf{z}^{\prime} \mathbf{z}\right)^{-1} \mathbf{z}^{\prime} \mathbf{W} \mathbf{z}
$$

É fácil verificar que, a estatística de Moran nada mais é que o coeficiente de inclinação da regressão $\mathbf{W z}$ contra $\mathbf{z}$, estimada por mínimos quadrados ordinários. Dada essa definição é tentador usar o teste- $t$ de significância do parâmetro para verificar a significância da estatística I de Moran, mas segundo Anselin (1993) esse procedimento não é apropriado.

De acordo com Gallo e Ertur (2003) a estatística $I$ de Moran dá a indicação formal de uma associação linear entre a variável de interesse subtraída da média (z) em relação a ela mesma defasada espacialmente (Wz). Segundo Pimentel e Haddad (2004, p. 9) “o resultado desta estatística indica a existência de autocorrelação positiva (negativa) caso seja maior

\footnotetext{
${ }^{4}$ Para maiores detalhes sobre a construção das diversas matrizes de pesos espaciais ver Anselin (1988), Tyszler (2006) e Almeida (2004).

${ }^{5} \mathbf{z}=(\mathbf{y}-\overline{\mathbf{y}})$
} 
(menor) do que sua esperança matemática" ${ }^{6}$. A esperança matemática indica o valor que seria esperado da estatística calculada sem haver estrutura de autocorrelação correlação espacial nos dados. Assim, se a estatística de $I$ for diferente da esperança de $I$ há autocorrelação espacial nos dados.

Como se trata de uma estatística é necessário verificar sua significância. Isso pode ser feito por meio de um teste de hipóteses, onde a hipótese nula é de ausência de autocorrelação espacial e a hipótese alternativa é a presença de autocorrelação espacial. Para isso, supõe-se que a estatística $I$ tenha uma distribuição de probabilidades normal ${ }^{7}$, e se a estatística estiver fora de um determinado nível crítico rejeita-se a hipótese nula. Como regra de decisão usa-se mais comumente o pseudo valor-p, que indica a probabilidade de se rejeitar a hipótese nula sendo ela verdadeira. Se o pseudo valor-p for menor que determinado nível de significância rejeita-se a hipótese nula.

\subsubsection{Diagrama de Dispersão de Moran}

Uma forma de visualizar essa estatística é por meio do diagrama de dispersão de Moran (Moran scatterplot), no qual a variável no eixo das abscissas é a variável em análise (y) e a variável no eixo das ordenadas é o cálculo da variável em análise defasada espacialmente $(\mathbf{W y})$. Considerando a variável em análise (y) como desvios em relação à sua média (z), é possível traçar quatro quadrantes (Alto-Alto, Baixo-Baixo, Alto-Baixo e BaixoAlto), conforme a Figura 2.

O primeiro quadrante mostra as regiões que apresentam valores acima da média para a variável em análise, cercadas por regiões que também apresentam valores acima da média para a variável em análise. Esse quadrante é classificado como alto-alto (AA). O segundo quadrante mostra as regiões com valores abaixo da média, cercados por vizinhos que apresentam valores acima da média. Este quadrante é geralmente classificado como baixo-alto (BA). O terceiro quadrante é constituído pelas regiões com valores abaixo da média para as variáveis em análise, cercados por vizinhos que também apresentam valores abaixo da média. Esse quadrante é classificado como baixo-baixo (BB). O quarto quadrante é formado pelas

${ }^{6}$ A esperança matemática do $I$ de Moran é dada pela expressão: $E(I)=\frac{-1}{(n-1)}$

${ }^{7}$ Essa distribuição pode ser construída através do pressuposto da aleatorização. O pressuposto da aleatorização, segundo Almeida (2004), assume que o mecanismo estocástico gerador dos dados é aleatório e o padrão dos dados observados é simplesmente um de muitas possíveis realocações das $n$ observações em $n$ locações. Primeiramente os valores observados para uma variável são aleatoriamente realocados (embaralhados) para as diversas regiões, depois, a estatística do teste é calculada para uma dessas realocações (embaralhamentos). Assim, obtém-se uma distribuição de referência empírica a partir dos cálculos da estatística para as realocações. 
regiões com valores acima da média para as variáveis em análise, cercados por regiões com valores abaixo da média. Esse quadrante é classificado como alto-baixo (AB) (PEROBELLI, et. al., 2007).

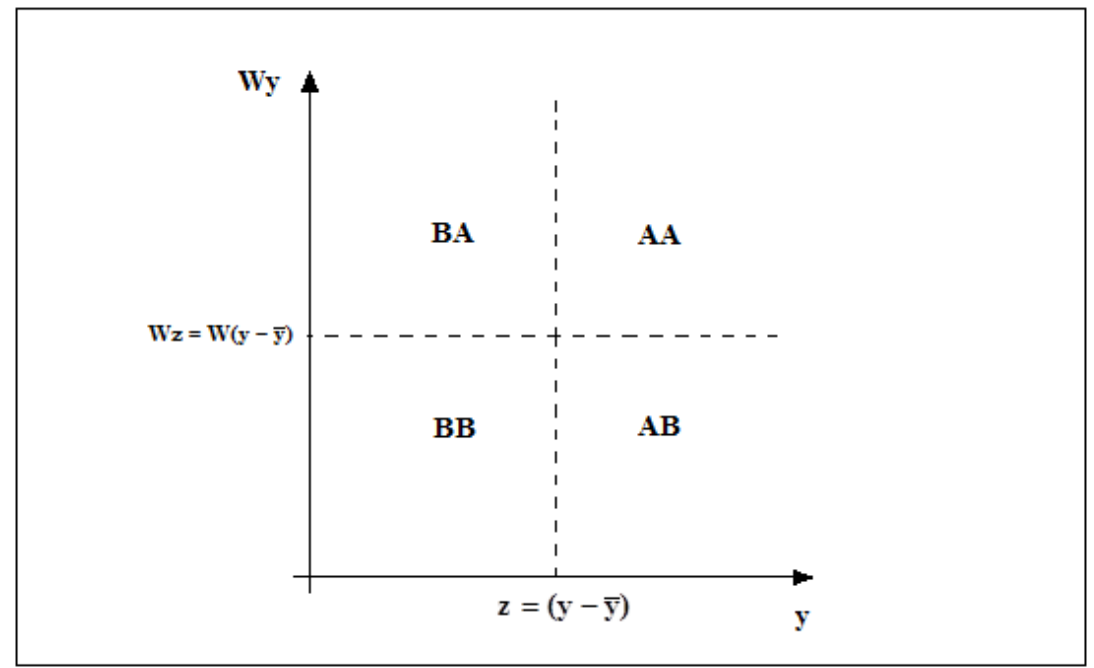

Figura 2 - Ilustração do diagrama de dispersão de Moran.

Fonte: Elaborado pelos autores.

Gallo e Ertur (2003) afirmam que os quadrantes AA e BB se referem a uma autocorrelação espacial positiva indicando clusters espaciais de valores semelhantes, e os quadrantes $\mathrm{BA}$ e $\mathrm{AB}$ aludem a uma autocorrelação negativa indicando clusters espaciais de valores não semelhantes.

\subsubsection{LISA}

Apesar do I de Moran oferecer a observação da existência de regimes espaciais globais nos dados, isso não responde outras questões, como: Uma indicação de ausência de autocorrelação global oculta padrões de associação local? Ou, uma forte indicação de autocorrelação global pode camuflar padrões locais de associação (clusters ou outliers espaciais). É necessário conhecer o local onde os padrões espaciais ocorrem. (PIMENTEL e HADDAD, 2004; ALMEIDA 2004).

Para superar essa dificuldade, Anselin (1995) propôs a estatística LISA (Local Indicators of Spatial Association) que precisa satisfazer dois critérios: primeiro, para cada observação o LISA deve oferecer uma indicação de clusters espaciais significantes de valores similares em torno da observação; segundo, a soma dos LISA's para todas as observações é proporcional ao indicador de associação espacial global. Formalmente, segundo Anselin (1995), tem-se: 


$$
I_{i}=z_{i} \sum_{j=1}^{n} w_{i j} z_{j}
$$

Onde $z_{i}$ e $z_{j}$ são desvios da variável de interesse em relação à média, e o somatório sobre $j$ é tal que somente os valores vizinhos diretos de $j$ são incluídos no cálculo da estatística.

O resultado desta estatística indica a existência de autocorrelação espacial local positiva (negativa), caso seja maior (menor) do que sua esperança matemática ${ }^{8}$. Segundo Perobelli et. al. (2007), a estatística pode ser interpretada da seguinte forma: valores positivos para $I_{i}$, indicam que existem clusters espaciais com valores similares; e valores negativos para $I_{i}$, indicam que existem clusters espaciais com valores diferentes entre os municípios e os seus vizinhos.

Como se trata de uma estatística, assim como o I de Moran, é necessário verificar sua significância. Nesse caso, a hipótese nula é de ausência de autocorrelação espacial local, enquanto a hipótese alternativa é de presença de autocorrelação espacial local. Para se construir a distribuição de probabilidades do LISA usa-se, também, o pressuposto da aleatorização, onde são auferidos pseudoníveis de significância.

Para esta análise foi utilizado o software GeoDa, desenvolvido Laboratório de Análises Espaciais da Universidade de Illinois - USA.

\subsection{Base de Dados}

O estudo utiliza os dados do Índice FIRJAN de Desenvolvimento Municipal (IFDM), calculado pela Federação das Indústrias do Rio de Janeiro (FIRJAN), abrangendo todos os municípios do Paraná, para os anos de 2000, 2005 e 2010. O índice tem periodicidade anual e é calculado para todos os municípios brasileiros, ele varia de 0 a 1 , em que, quanto mais próximo de 1 mais desenvolvido é o município. Justifica-se a utilização do IFDM como medida de desenvolvimento, pois as fontes de dados do IFDM são oficiais e o mesmo segue a metodologia do IDH - Índice de Desenvolvimento Humano da ONU -, sua metodologia permite a comparação quantitativa serial e temporal dos municípios analisados, ademais, o índice consolidado é desagregado para Emprego \& Renda, Educação e Saúde.

O IFDM é um indicador multidimensional, que abrange três dimensões importantes

\footnotetext{
${ }^{8}$ Sob o pressuposto da aleatorização, o valor esperado da estatística $I_{i}$ é dado por: $E\left(I_{i}\right)=\frac{-w_{i}}{(n-1)}$, em que $w_{i}$ é a soma dos elementos da linha.
} 
para o desenvolvimento: Emprego \& Renda, Educação e Saúde. Cada área é composta por indicadores simples ${ }^{9}$ :

Tabela 1 - Indicadores utilizados no IFDM e suas respectivas variáveis.

\begin{tabular}{lll}
\hline \multicolumn{1}{c}{ Emprego \& Renda } & \multicolumn{1}{c}{ Educação } & \multicolumn{1}{c}{ Saúde } \\
Geração de emprego & $\begin{array}{l}\text { Taxa de abandono } \\
\text { Taxa de distorção idade- Óbitos infantis por causas } \\
\text { Estoque de emprego formal } \\
\text { série }\end{array}$ & $\begin{array}{l}\text { Numero de consultas pré-natal } \\
\text { Salários médios do emprego } \\
\text { formal }\end{array}$ \\
& $\begin{array}{l}\text { Percentual de docentes } \\
\text { com ensino superior } \\
\text { Média de horas aula por causa não definidas } \\
\text { diária } \\
\text { Resultado do IDEB }\end{array}$ \\
\hline
\end{tabular}

Fonte: FIRJAN, 2012.

As três dimensões são consideradas os pilares para o desenvolvimento de uma determinada localidade. A dimensão Emprego \& Renda tem por objetivo captar as características econômicas dos municípios, acompanhando a movimentação e as características do mercado formal de trabalho, com base nos dados disponibilizados pelo Ministério do Trabalho. O IFDM Educação visa captar tanto a oferta como a qualidade da educação do ensino fundamental e pré-escola. A dimensão Saúde procura verificar desempenho dos municípios com relação à saúde básica.

\section{Resultados e Discussão}

Nesta seção são descritos os resultados obtidos por meio da Análise Exploratória de Dados Espaciais para os dados do IFDM, para o período proposto. Primeiramente, apresentase uma análise descritiva para o IFDM, para os anos de 2000, 2005 e 2010, em seguida, é analisado o I de Moran global e seu diagrama de dispersão e, na sequência, são apresentados os resultados do I de Moran local.

\footnotetext{
${ }^{9}$ Segundo Jannuzzi (2009), os indicadores compostos, também chamados de indicadores sintéticos ou índices sociais, são elaborados mediante a aglutinação de dois ou mais indicadores simples, referidos a uma mesma ou diferentes dimensões da realidade social.
} 


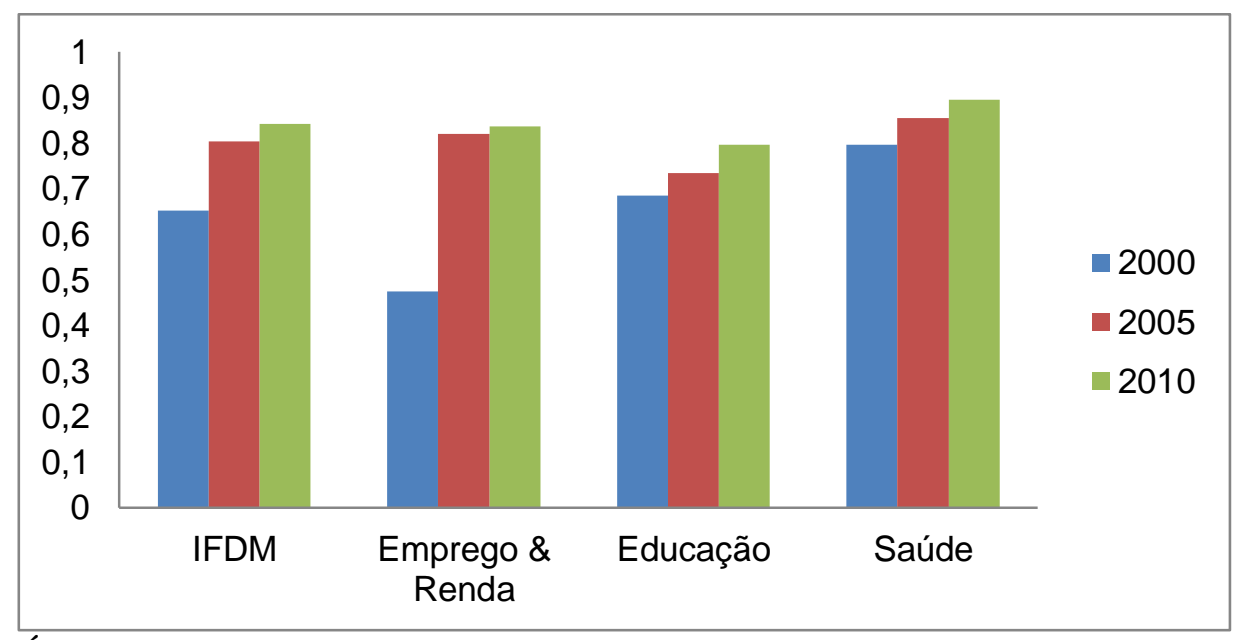

Figura 3 - Índice Firjan de Desenvolvimento Municipal - IDFM - para o Estado do Paraná Fonte: Elaborado pelos autores a partir dos dados da FIRJAN.

A Figura 3 apresenta a evolução dos valores do índice FIRJAN para o Estado do Paraná, referente ao período analisado. Observa-se que, tanto o índice geral (IFDM) quanto suas dimensões (Emprego \& Renda, Educação, e Saúde) expressam uma melhoria na década de 2000. De 2000 a 2010 o IFDM apresentou uma variação positiva de 29\%, sendo o IFDM Emprego \& Renda o índice que apresentou maior variação; em 2010 essa dimensão foi 76\% maior que em 2000.

\section{"I" de Moran}

A primeira etapa para a análise de presença de autocorrelação espacial nos dados é o índice $I$ de Moran. Uma autocorrelação espacial positiva indica que os municípios que possuem um alto índice de desenvolvimento são vizinhos de outros municípios com alto índice de desenvolvimento, e municípios com baixo desenvolvimento são circundados por outros municípios também ostentando baixo desenvolvimento.

Tabela 2 - I de Moran, média, desvio padrão e pseudo valor-p para o IFDM, IFDM - Emprego \& Renda, IFDM - Educação e IFDM - Saúde para os anos de 2000, 2005 e 2010.

\begin{tabular}{|c|c|c|c|c|}
\hline & I de Moran & Média & Desvio Padrão & Pseudo valor-p \\
\hline & \multicolumn{4}{|c|}{ IFDM } \\
\hline 2000 & 0,3817 & $-0,0021$ & 0,0310 & 0,001 \\
\hline 2005 & 0,3533 & $-0,0012$ & 0,0303 & 0,001 \\
\hline \multirow[t]{2}{*}{2010} & 0,2919 & $-0,0028$ & 0,3070 & 0,001 \\
\hline & \multicolumn{4}{|c|}{ IFDM - Emprego \& Renda } \\
\hline 2000 & 0,0825 & $-0,0027$ & 0,0297 & 0,006 \\
\hline 2005 & 0,2076 & $-0,0021$ & 0,0304 & 0,001 \\
\hline \multirow[t]{2}{*}{2010} & 0,2005 & $-0,0013$ & 0,0317 & 0,001 \\
\hline & \multicolumn{4}{|c|}{ IFDM - Educação } \\
\hline 2000 & 0,5139 & $-0,0034$ & 0,0303 & 0,001 \\
\hline 2005 & 0,4567 & $-0,0037$ & 0,0302 & 0,001 \\
\hline \multirow[t]{2}{*}{2010} & 0,4086 & $-0,0029$ & 0,0317 & 0,001 \\
\hline & \multicolumn{4}{|c|}{ IFDM - Saúde } \\
\hline 2000 & 0,3598 & $-0,0022$ & 0,0305 & 0,001 \\
\hline 2005 & 0,3705 & $-0,003$ & 0,0319 & 0,001 \\
\hline 2010 & 0,3239 & $-0,0036$ & 0,0316 & 0,001 \\
\hline
\end{tabular}

Fonte: Elaborado pelos autores a partir dos dados da FIRJAN.

Nota: A pseudo-significância empírica é baseada em 999 permutações aleatórias. 
A Tabela 3 mostra os resultados do I de Moran, média, desvio padrão e pseudo valorp para o IFDM e suas respectivas dimensões, para os anos de 2000, 2005 e 2010. Nota-se que, como o $I$ de Moran para todas as variáveis e anos é maior que a sua esperança matemática ${ }^{10}$, existe uma autocorrelação espacial positiva nos dados. Os valores são estatisticamente significativos, com pseudo-p $(0,001)$.

\section{Diagrama de Dispersão de Moran}

A segunda etapa é a análise do diagrama de dispersão. As Figuras 4, 5, 6 e 7 apresentam o diagrama de dispersão para as variáveis analisadas. No eixo das abscissas estão o IFDM e suas dimensões, para os anos em análise, e no eixo das ordenadas apresentam-se as mesmas variáveis, só que defasadas espacialmente. A reta representa a tendência central do agrupamento dos dados, sua inclinação é justamente o valor do $I$ de Moran para cada ano. Se a inclinação da reta for positiva, existe autocorrelação espacial positiva. Se a inclinação da reta for negativa, haverá autocorrelação negativa.

Corroborando os resultados da Tabela 2, primeiramente, é possível perceber que todas as Figuras (Figuras 4, 5, 6 e 7) possuem uma reta com inclinação positiva, indicando uma autocorrelação espacial positiva nos dados. Em segundo lugar, observa-se que a maioria dos municípios está localizada nos quadrantes alto-alto (AA) e baixo-baixo (BB), em todas as variáveis e para todos os anos avaliados.

O quadrante AA representa clusters de desenvolvimento, pois nesse quadrante existem municípios com nível de desenvolvimento acima da média, circundados por municípios com nível de desenvolvimento acima da média. Enquanto o quadrante BB representa clusters de subdesenvolvimento, onde há municípios com nível de desenvolvimento abaixo da média que tem a sua volta municípios com nível de desenvolvimento abaixo da média.

\footnotetext{
${ }^{10}$ Lembrando que a esperança matemática do I de Moran é dada pela expressão: $E(I)=\frac{-1}{(n-1)}$. Como $n=399$, que é o total de municípios, substituindo na expressão temos que: $E(I)=-0,0025$.
} 


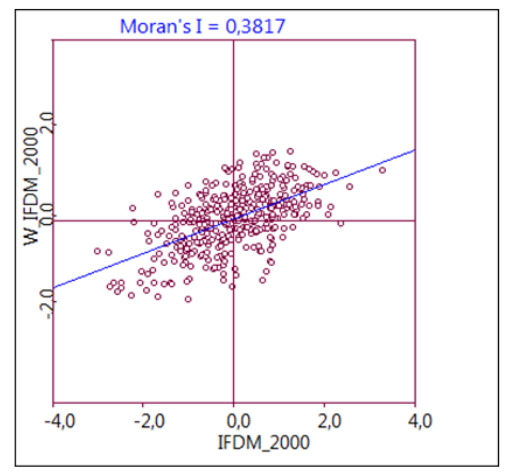

2000

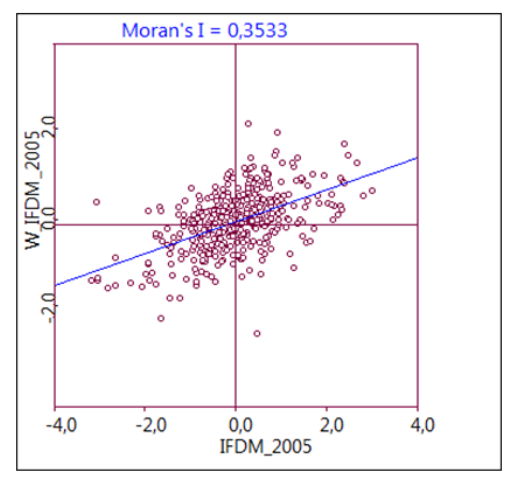

2005

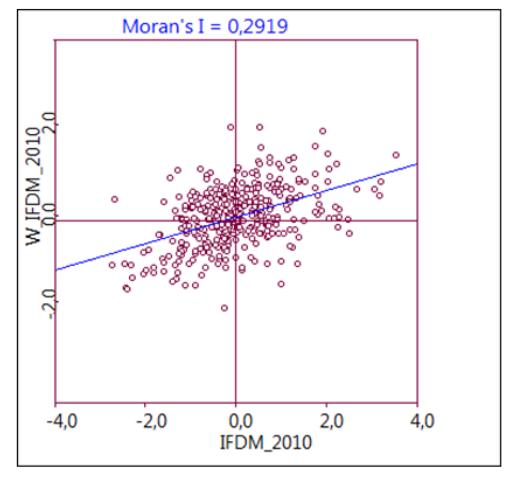

2010

(A)

(B)

(C)

Figura 4 - Diagrama de dispersão de Moran para o IFDM, Paraná, 2000, 2005 e 2010. Fonte: Elaborado pelos autores a partir dos dados da FIRJAN.

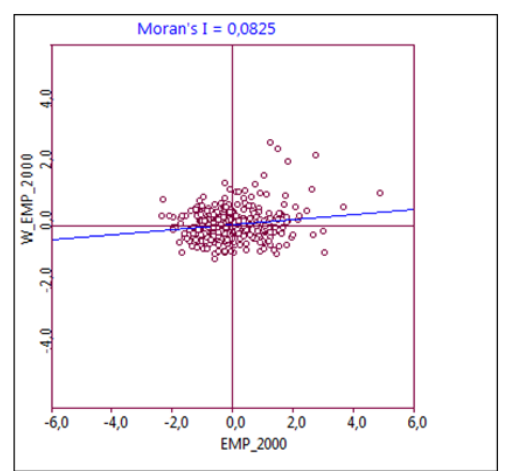

2000

(A)

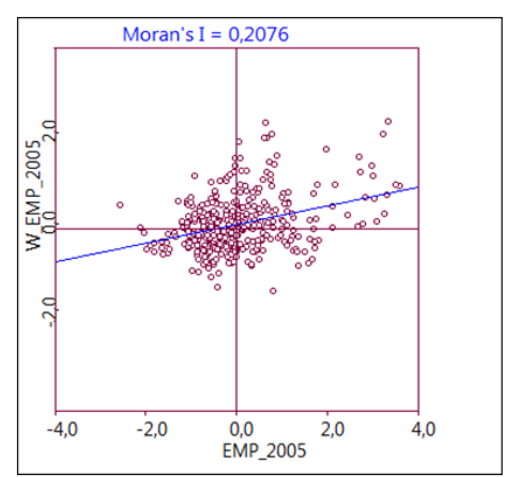

2005

(B)

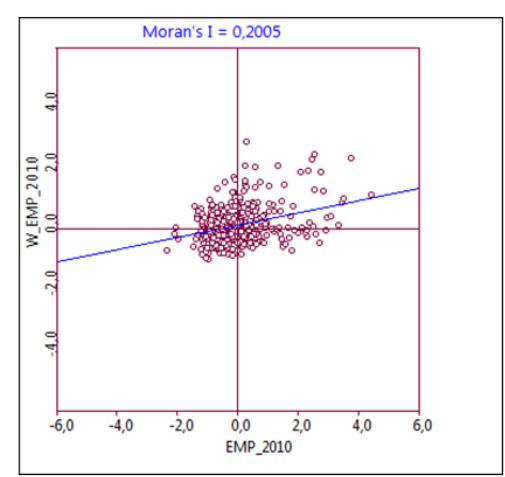

2010

(C)

Figura 5 - Diagrama de dispersão de Moran para o IFDM - Emprego \& Renda, Paraná, 2000, 2005 e 2010.

Fonte: Elaborado pelos autores a partir dos dados da FIRJAN.

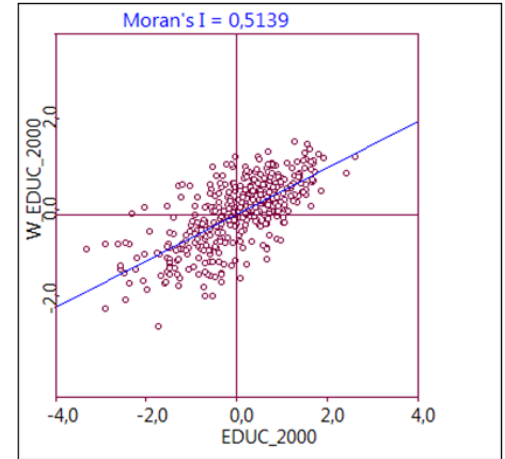

2000

(A)

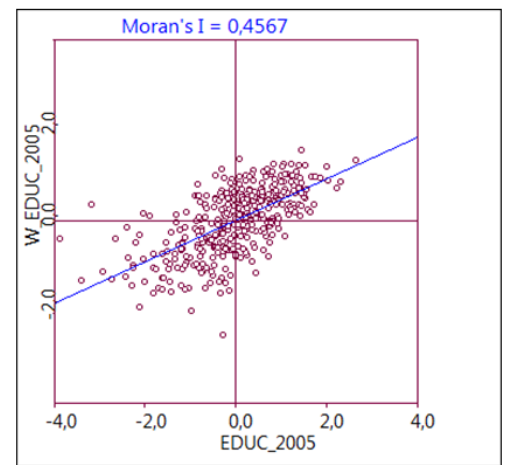

2005

(B)

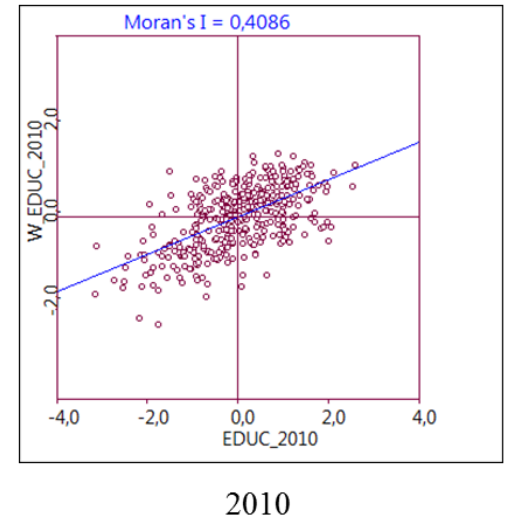

(C)

Figura 6 - Diagrama de dispersão de Moran para o IFDM - Educação, Paraná, 2000, 2005 e 2010.

Fonte: Elaborado pelos autores a partir dos dados da FIRJAN. 


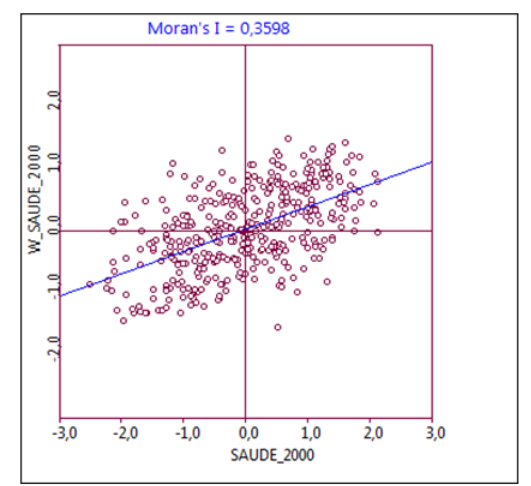

2000

(A)

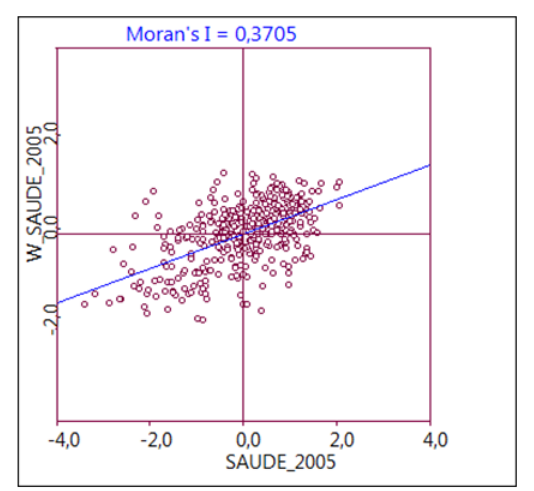

2005

(B)

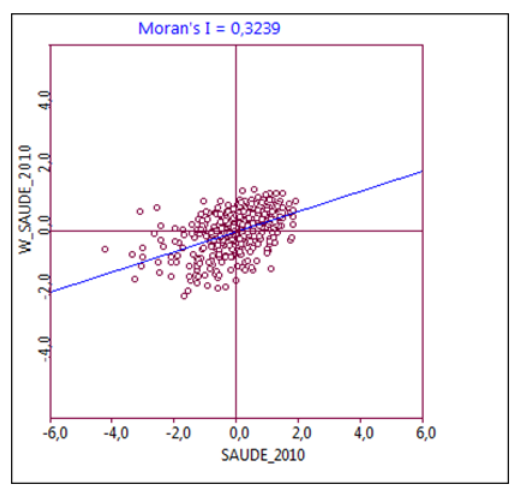

2010

(C)

Figura 7 - Diagrama de dispersão de Moran para o IFDM - Saúde, 2000, 2005 e 2010.

Fonte: Elaborado pelos autores a partir dos dados da FIRJAN.

Outro ponto interessante a se observar, é que há uma diminuição na autocorrelação espacial do IDFM ao longo da última década. O I de Moran caiu de 0,3817 para 0,2919. Assim, com base no índice $I$ de Moran para as demais dimensões que compõem o IFDM, é possível afirmar que essa queda na concentração espacial está sendo causada, em grande parte, pelo IFDM - Educação.

\section{Indicadores Locais de Associação Espacial (LISA)}

Segundo Almeida (2004) o problema com os diagramas de dispersão de Moran é que eles exibem clusters tanto estatisticamente significantes quanto não. Não há sentido levar em conta a análise de clusters que não sejam estatisticamente significantes. Para contornar tal problema, foi construída a estatística LISA, que avalia o padrão de associação espacial local a determinados níveis de significância. Para cada município é computado um $I_{i}$, com seu respectivo nível de significância. Tamanha quantidade de informação pode confundir o pesquisador, se colocada em tabelas. Uma forma mais eficiente de apresentar esse conjunto de estatísticas é mapeá-las.

Os mapas 1, 2, 3 e 4 são os chamados mapas de clusters espaciais. Eles são a combinação da informação do diagrama de dispersão de Moran e a informação de significância das medidas de associação local $I_{i}$. Tais mapas ilustram a classificação das quatro categorias de associação espacial que são estatisticamente significantes.

No Mapa 1 é possível identificar clusters de desenvolvimento e de subdesenvolvimento no estado do Paraná ao longo da última década. Nos três anos 
analisados, os clusters de desenvolvimento se distribuíram basicamente em três mesorregiões: Norte Central, Oeste, e Metropolitana de Curitiba. No que tange aos clusters de subdesenvolvimento, no ano de 2000 eles se distribuíram com maior predominância nas mesorregiões Centro Sul, Centro Oriental e norte da mesorregião Metropolitana de Curitiba. No ano de 2005, os clusters predominaram na mesorregião Centro Sul, e em uma região que compreende o sul da mesorregião Norte Central e o oeste da mesorregião Centro Oriental. No ano de 2010 se manteve praticamente a mesma configuração do ano de 2005, mas agora com um cluster em uma região que abarca norte da mesorregião Metropolitana de Curitiba e o leste da mesorregião Centro Oriental.

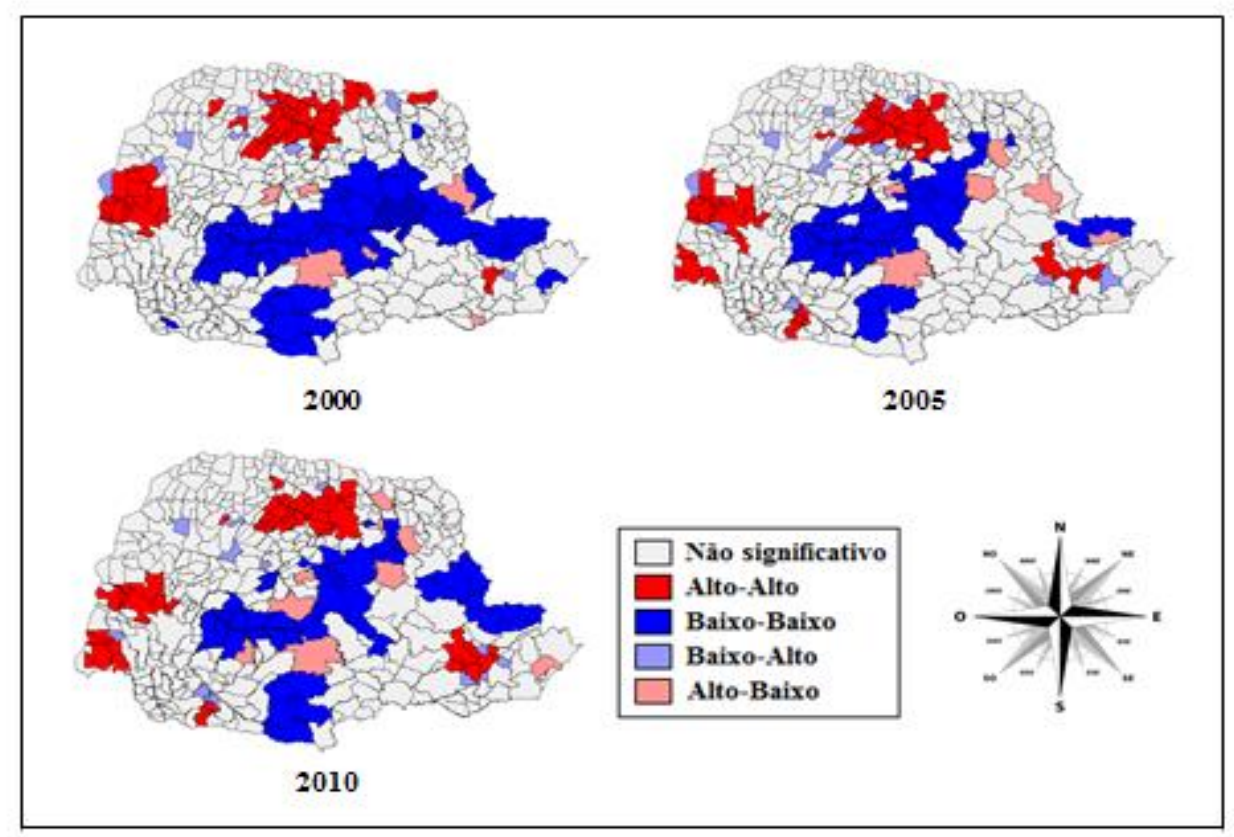

Mapa 1 - Clusters, IFDM, Paraná - 2000, 2005 e 2010.

Fonte: Elaborado pelos autores a partir dos dados da FIRJAN.

Quando analisada apenas a dimensão Emprego e Renda (Mapa 2), é possível visualizar que no ano de 2000 existiam basicamente dois clusters AA, um na mesorregião Metropolitana de Curitiba e outro na mesorregião Sudeste. Em 2005, verifica-se um cluster AA que compreendia as mesorregiões Metropolitana de Curitiba e Centro Oriental, além de clusters nas mesorregiões Norte Central e Oeste, os clusters BB se concentraram no meio do estado do Paraná. No ano de 2010, é mantida praticamente a mesma configuração. Os clusters $\mathrm{BB}$, nos três anos em questão se mantiveram no centro do estado. 


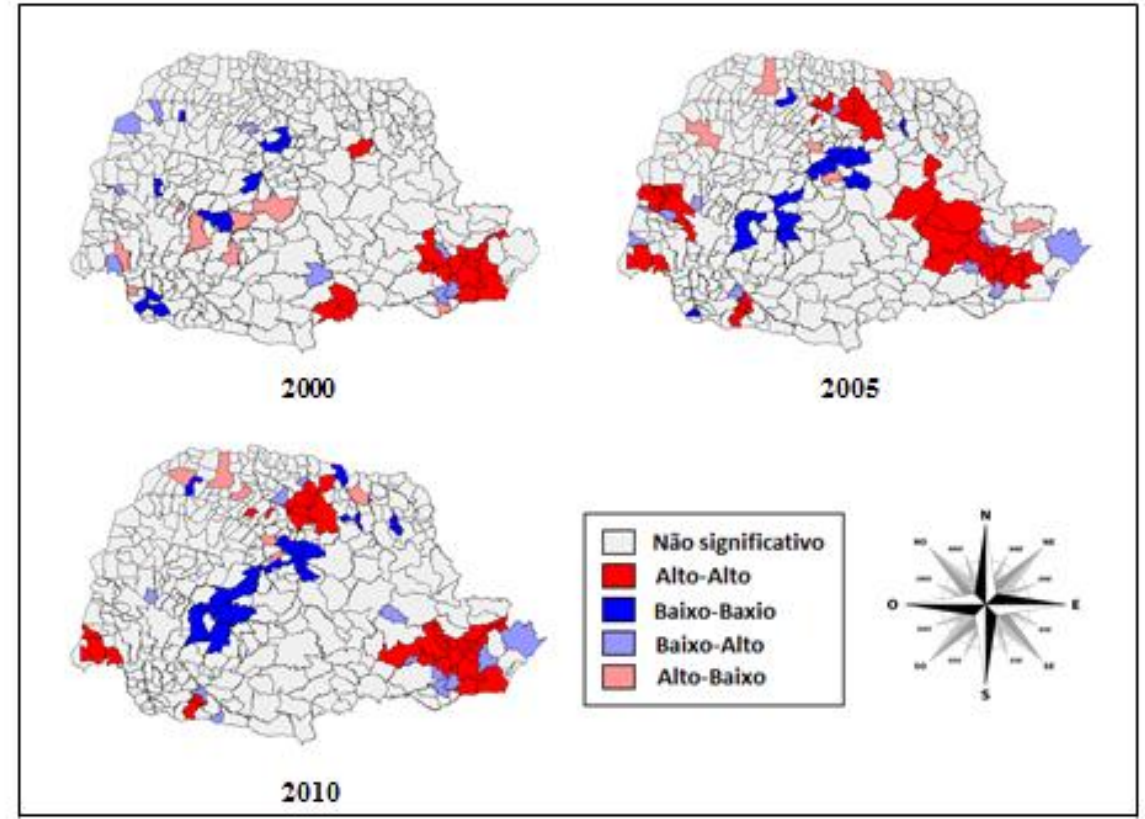

Mapa 2 - Clusters, IFDM - Emprego e Renda, Paraná - 2000, 2005 e 2010.

Fonte: Elaborado pelos autores a partir dos dados da FIRJAN.

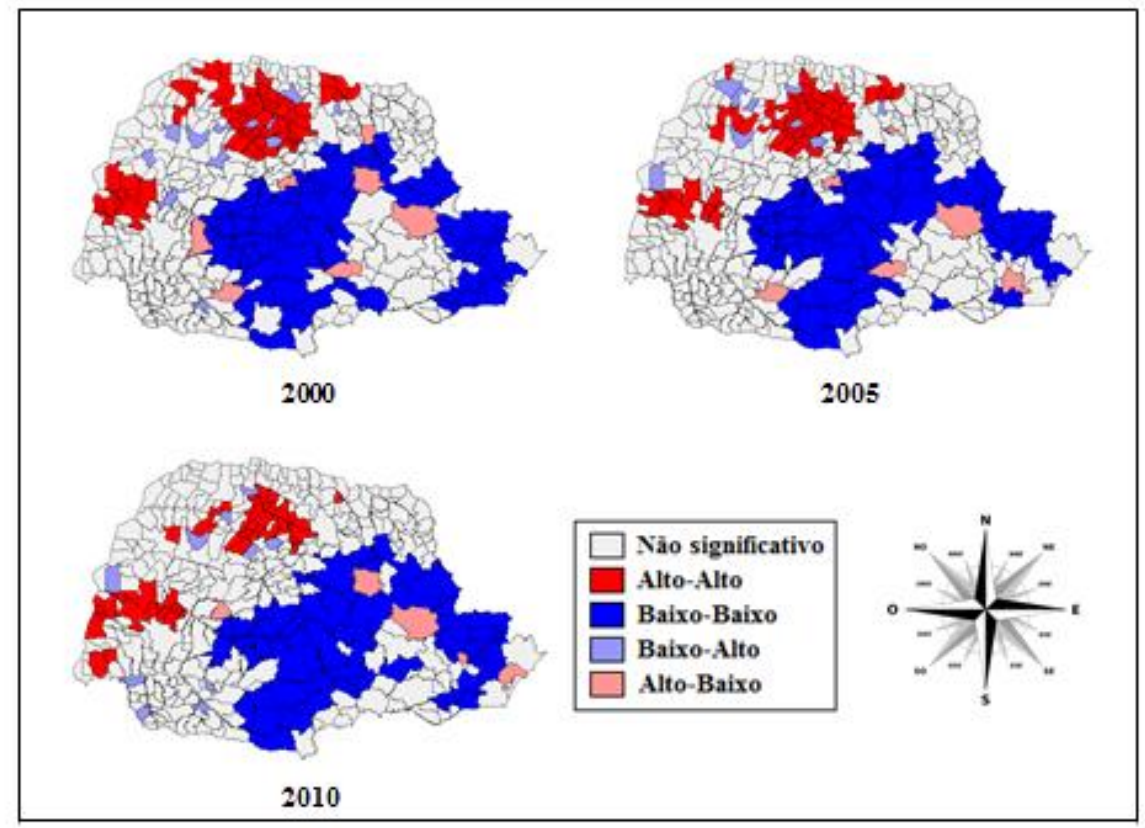

Mapa 3 - Clusters, IFDM - Educação, Paraná - 2000, 2005 e 2010.

Fonte: Elaborado pelos autores a partir dos dados da FIRJAN. 


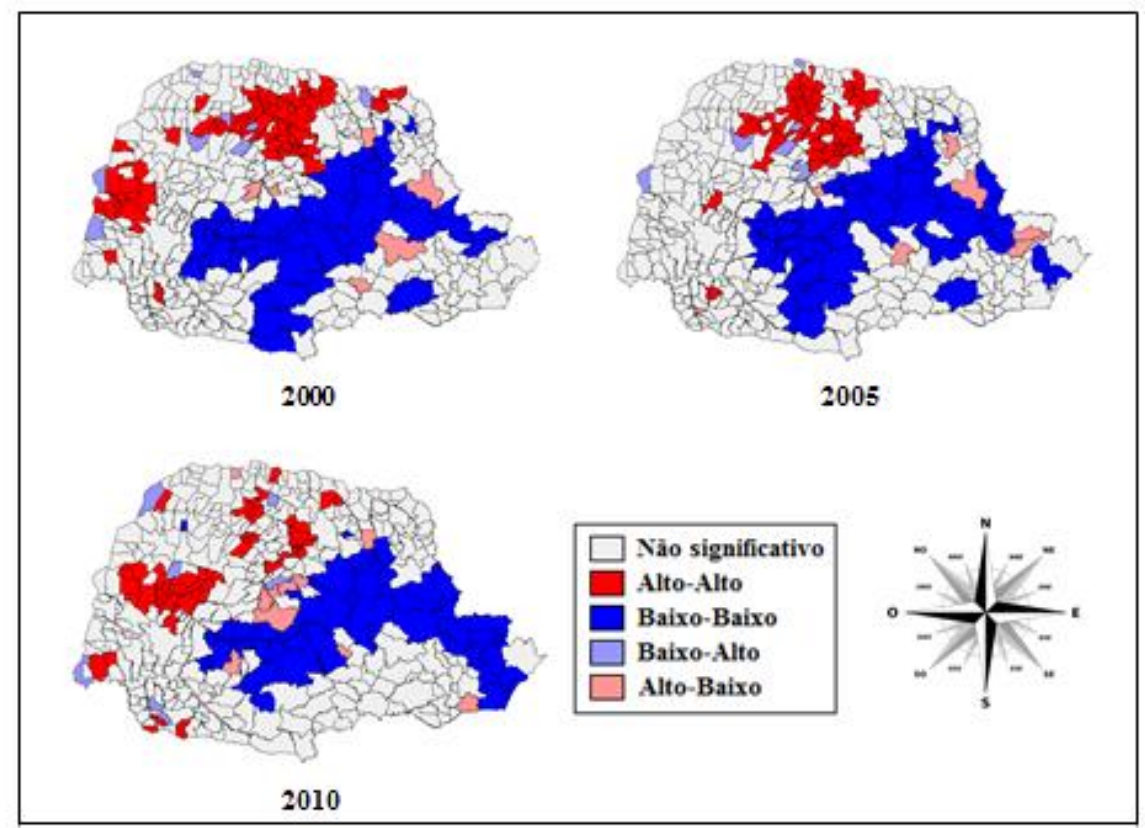

Mapa 4 - Clusters, IFDM - Saúde, Paraná - 2000, 2005 e 2010. Fonte: Elaborado pelos autores a partir dos dados da FIRJAN.

No que se refere à dimensão Educação (Mapa 7), nos três anos analisados é possível identificar dois clusters AA nas mesorregiões Norte Central, Noroeste e Oeste, e um cluster BB que ocupa praticamente toda a região central e leste do estado. A dimensão Saúde (Mapa 8) apresenta esse mesmo padrão de clusters BB, e com relação aos clusters AA, em 2000, eles se concentraram nas mesorregiões Norte Central, Noroeste e Oeste. Em 2005, existia apenas um cluster na mesorregião Norte Central e em 2010, existia um cluster que compreendia as regiões Oeste e Centro Ocidental e pequenos clusters na mesorregião Norte Central.

\section{Considerações finais}

No presenta trabalho foi possível constatar que o índice FIRJAN para o estado do Paraná, tanto o índice consolidado (IFDM) quanto suas dimensões (Emprego \& Renda, Educação, e Saúde) expressaram uma melhoria na década de 2000. Tal fato sinaliza uma evolução positiva nos indicadores e, portanto, padrão desenvolvimento do estado na última década.

No que tange à análise espacial e verificação de autocorrelação nos dados, constatouse a existência de autocorreção espacial positiva, para todos os anos estudados. Isso demonstra que existem concentrações espaciais, também chamados de clusters espaciais. Esses clusters se dividem em dois grupos: de alto desenvolvimento e de baixo desenvolvimento. 
No período analisado, os clusters de desenvolvimento se distribuíram basicamente em três mesorregiões: Norte Central, Oeste, e Metropolitana de Curitiba. Os clusters de subdesenvolvimento se distribuíram na região central e leste do estado. A partir das dimensões Educação e Saúde, observou-se uma forte tendência de concentração de subdesenvolvimento na região central e leste do estado do Paraná, incluindo a mesorregião Metropolitana de Curitiba. Os clusters de desenvolvimento, considerando Educação e Saúde, se concentraram predominantemente nas mesorregiões Norte Central, Noroeste e Oeste. Em relação à dimensão Emprego \& Renda do desenvolvimento, houve uma concentração peculiar, identificou-se um clusters AA em uma região que compreendia as mesorregiões Metropolitana de Curitiba e Centro Oriental, além de clusters nas mesorregiões Norte Central e Oeste. Os clusters BB se concentraram na região central do estado.

A principal contribuição deste estudo foi o aprofundamento na compreensão do padrão de desenvolvimento do estado do Paraná e a análise em termos regionais, ou seja, o padrão espacial do Indicador IFDM. Verificou-se que na última década ocorreu uma evolução positiva no padrão de desenvolvimento do Paraná, todavia, quando analisado em termos regionais e o resultado é que esse padrão não foi idêntico para todas as regiões. O estudo permitiu identificar e analisar o padrão de desenvolvimento municipal paranaense e a dinâmica de formação de clusters de desenvolvimento/subsenvolvimento. O estudo identificou assimetrias a partir dos indicadores de desenvolvimento municipais paranaenses, concentrando nas mesorregiões central e leste do estado clusters de subsenvolvimento.

Para estudos futuros, lança-se o desafio de compreender os principais fatores de influência nos indicadores de desenvolvimento das regiões com clusters de desenvolvimento, sendo que devem nortear as políticas para as regiões com baixo grau de desenvolvimento.

\section{Referências}

ALMEIDA, E. Curso de Econometria Espacial Aplicada. ESALQ-USP: Piracicaba, 2004.

ANSELIN, L. Spatial Econometrics: methods and models. Boston: Kluwer Academic, 1988.

ANSELIN, L. The Moran scatterplot as an ESDA tool to assess local instability in spatial association. GISDATA Specialist Meeting on GIS and Spatial Analysis, December 1-5, 1993.

ANSELIN, L. Local Indicators of Spatial Association - LISA. Geographical Analysis, 27, n.2, p. 93-115, 1995. 
ANSELIN, L.; SYABRI, I; KHO, Y. GeoDa: An Introduction to Spatial Data Analysis. Geographical Analysis 38 (1), 5-22. 2006.

COSTA, A.M.B., SILVA, F.M., GOMES, C., CUELlAR, M.Z., ALMEIDA, S.A.S., AMORIN, R. F., CARVALHO, M.J.M. Comportamento espacial do índice de desenvolvimento humano no Rio Grande do Norte com o uso do programa TerraView (desenvolvido pelo INPE). Anais. XIII Simpósio Brasileiro de Sensoriamento Remoto, Florianópolis, Brasil, 21-26 abril 2007, INPE, p. 5159-5166.

FIRJAN. Índice Firjan de Desenvolvimento Municipal IFDM. Ano base 2010. Disponível em:

http://www.firjan.org.br/lumis/portal/file/fileDownload.jsp?fileId=2C908CEC3B4DD6E3013 B534D16FD14A7. Acesso em maio de 2013.

GALLO, J. L.; ERTUR, C. Exploratory spatial data analysis of the distribution of regional per capita GDP in Europe, 1980-1995. Regional Science. n. 82, p.175-201, 2003.

GAMA, R. G., STRAUCH, J. C. M. Análise espacial de indicadores de Desenvolvimento Sustentável: Aplicação do índice de Moran, Proceedings os 12 Congresso de Geógrafos de America Latina, Montevidéu, 2009.

GEARY, R. C. The contiguity ratio and statistical mapping. The Incorporated Statistician. v. 05, n. 03, p. 115-145, nov 1954.

JANNUZZI. P. M. Indicadores sociais no Brasil: conceitos fontes de dados e aplicações. $4^{\text {a }}$ ed. Campinas: Alínea, 2009.

LEITE, P. S. Novo enfoque do desenvolvimento econômico e as teorias convencionais. Fortaleza: Imprensa Universitária, 1983.

LORENA, R. B.; BERGAMASCHI, R. B.; LEITE, G. R. Análise Exploratória Espacial do Índice de Desenvolvimento Humano Municipal do Estado do Espírito Santo. Anais. XV Simpósio Brasileiro de Sensoriamento Remoto - SBSR, Curitiba, PR, Brasil, 30 de abril a 05 de maio de 2011, INPE p.4776.

MORAN, P. A. P. The interpretation of Statistical Maps. Journal of the Royal Statistical Society, Series B, vol. 10, n. 2, p. 243-251, 1948.

PEROBELLI, F. S.; ALMEIDA, E. S.; ALVIN, M. I. S.; FERREIRA, P. G. C. Produtividade do setor agrícola brasileiro (1991-2003): uma análise espacial. Nova Economia, Belo Horizonte, vol. 17, n. 1, p.65-91, jan/abr 2007.

PIMENTEL, E. A.; HADDAD, E. A. Análise da distribuição espacial da renda no estado de minas gerais: uma abordagem setorial. Texto para discussão, NEREUS. São Paulo, 2004.

TYSZLER, M. Econometria espacial: discutindo medidas para a matriz de ponderação espacial. Dissertação de Mestrado. Fundação Getúlio Vargas - Escola de Administração de Empresas de São Paulo. São Paulo, 2006. 
VALE, F. F. R.; SILVA, J. L. M. Rurality in Brazilian Northeast: spatial distribution and cluster identification. Wye City Group on Statistics on Rural Development and Agriculture Household Income, Rio de Janeiro 9-1 ${ }^{\text {th }}$ November 2011. 